\title{
Soliton Solutions of a General Rosenau-Kawahara-RLW Equation
}

\author{
Jin-ming Zuo ${ }^{1}$ \\ ${ }^{1}$ School of Science, Shandong University of Technology, Zibo 255049, PR China \\ Correspondence: Jin-ming Zuo, School of Science, Shandong University of Technology, Zibo 255049, PR China. \\ E-mail: zuojinming@ sdut.edu.cn
}

Received: February 3, 2015 Accepted: February 22, 2015 Online Published: March 22, 2015

doi:10.5539/jmr.v7n2p24 URL: http://dx.doi.org/10.5539/jmr.v7n2p24

\begin{abstract}
In this paper, we consider a general Rosenau-Kawahara-RLW equation. The exact bright and dark soliton solutions for the considered model are obtained by sech and tanh ansatzes methods. The mass and momentum conserved quantities are also calculated for the case of bright soliton solution.
\end{abstract}

Keywords: bright and dark soliton solutions, Rosenau-Kawahara-RLW equation, sech and tanh ansatzes

\section{Introduction}

Nonlinear partial differential equations (PDEs) are special classes of mathematics and physics, which have been studied intensively in past several decades. It is well known that seeking traveling-wave solutions for PDEs, by using different methods, has long been a major concern for mathematicians, physicists, and engineers. However, the existence of soliton type solutions for PDEs is of particular interest because of their extensive applications in many physics areas such as nonlinear optics, plasmas, fluid mechanics, condensed matter, electro magnetics and many more. Most famous equations with soliton solutions include the nonlinear Schrödinger (NLS), Korteweg-de Vries (KdV) (Korteweg, \& Vires, 1895), Regularized Long Wave (RLW) (Benjamin, Bona, \& Mahony, 1972), sine-Gordon (sG) and Rosenau equation (Rosenau, 1988), and so on. In this paper, we will investigate soliton solutions of the following generalized Rosenau-Kawahara-RLW equation:

$$
u_{t}-\alpha u_{x x t}+\beta u_{x x x x t}+\gamma u_{x}+\delta u^{n} u_{x}+\varepsilon u_{x x x}+\lambda u_{x x x x x}=0 .
$$

where $\alpha, \beta, \gamma, \delta, \varepsilon$ and $\lambda$ are real valued constants while the parameter $n \neq 0$ dictates the power law nonlinearity, which includes many important cases below:

When $\alpha=\varepsilon=\lambda=0$, the equation (1) becomes the general Rosenau equation:

$$
u_{t}+\beta u_{x x x x t}+\gamma u_{x}+\delta u^{n} u_{x}=0 .
$$

When $\alpha=\beta=0$, the equation (1) becomes the general Kawahara (or fifth-order KdV) equation:

$$
u_{t}+\gamma u_{x}+\delta u^{n} u_{x}+\varepsilon u_{x x x}+\lambda u_{x x x x x}=0 .
$$

When $\beta=\varepsilon=\lambda=0$, the equation (1) becomes the general RLW equation:

$$
u_{t}-\alpha u_{x x t}+\gamma u_{x}+\delta u^{n} u_{x}=0 .
$$

and other cases refer (Zuo, 2009; Esfahani, 2011; Razborova, Ahmed, \& Biswas, 2014).

Our interest in this paper is to search for the soliton solutions for the equation (1). The technique that will be used is solitary wave ansatzes in the form of sech and tanh functions, which are one of the most effective direct methods to construct solitary wave solutions of PDEs, see (Esfahani, 2011; Razborova, Ahmed, \& Biswas, 2014; Triki, \& Wazwaz, 2009; Biswas, 2009) and references therein. 


\section{Sech Ansatz Method}

To obtain bright soliton solution of the equation (1), we assume a solitary wave ansatz of the form (Esfahani, 2011; Razborova, Ahmed, \& Biswas, 2014; Triki, \& Wazwaz, 2009; Biswas, 2009):

$$
u(x, t)=A \operatorname{sech}^{p} \xi .
$$

where $\xi=k(x-c t), A$ is the amplitude of the soliton and $k$ is the inverse widths of the solitary wave, and $c$ represents the velocity of the soliton and the exponent $p$ will be determined later. From (5), we obtain

$$
\begin{gathered}
u_{t}=A p k c \operatorname{sech}^{p} \xi \tanh \xi, \\
u_{x}=-A p k \operatorname{sech}^{p} \xi \tanh \xi \\
u^{n} u_{x}=-A^{n+1} p k \operatorname{sech}^{(n+1) p} \xi \tanh \xi \\
u_{x x t}=-A k^{3} c p(p+1)(p+2) \operatorname{sech}^{p+2} \xi \tanh \xi+A k^{3} c p^{3} \operatorname{sech}^{p} \xi \tanh \xi, \\
u_{x x x}=A k^{3} p(p+1)(p+2) \operatorname{sech}^{p+2} \xi \tanh \xi-A k^{3} p^{3} \operatorname{sech}^{p} \xi \tanh \xi \\
u_{x x x x t}=A k^{5} c p(p+1)(p+2)(p+3)(p+4) \operatorname{sech}^{p+4} \xi \tanh \xi \\
-2 A k^{5} c p(p+1)(p+2)\left(p^{2}+2 p+2\right) \operatorname{sech}^{p+2} \xi \tanh \xi \\
+A k^{5} c p^{5} \operatorname{sech}^{p} \xi \tanh \xi \\
u_{x x x x x}=-A k^{5} p(p+1)(p+2)(p+3)(p+4) \operatorname{sech}^{p+4} \xi \tanh \xi \\
+2 A k^{5} p(p+1)(p+2)\left(p^{2}+2 p+2\right) \operatorname{sech}^{p+2} \xi \tanh \xi \\
-A k^{5} p^{5} \operatorname{sech}^{p} \xi \tanh \xi
\end{gathered}
$$

Substituting (6)-(12) into (1) gives

$$
\begin{array}{r}
A k p\left[c-\gamma-p^{2} k^{2}(\alpha c+\varepsilon)+k^{4} p^{4}(\beta c-\lambda)\right] \operatorname{sech}^{p} \xi \tanh \xi \\
+A k^{3} p(p+1)(p+2)\left[\alpha c+\varepsilon+2 k^{2}\left(p^{2}+2 p+2\right)(\lambda-\beta c)\right] \operatorname{sech}^{p+2} \xi \tanh \xi \\
+A k^{5} p(p+1)(p+2)(p+3)(p+4)(\beta c-\lambda) \operatorname{sech}^{p+4} \xi \tanh \xi \\
-\delta A^{n+1} p k \operatorname{sech}^{(n+1) p} \xi \tanh \xi=0 .
\end{array}
$$

Equating the highest exponents of $\operatorname{sech}^{p+4} \xi \tanh \xi$ and $\operatorname{sech}^{(n+1) p} \xi \tanh \xi$ terms in (13), we get

$$
(n+1) p=p+4
$$

which yields the following analytical condition

$$
p=\frac{4}{n}
$$

Now, from (13), setting the coefficients of $\operatorname{sech}^{p+j} \xi \tanh \xi(j=0,2,4)$ to zero, we obtain bright soliton solution of the equation (1):

$$
u(x, t)=A \operatorname{sech}^{\frac{4}{n}}[k(x-c t)] .
$$

where $A=\left\{\frac{(n+1)(3 n+4)(n+4)\left[4 a(\lambda-\beta \gamma)\left(n^{2}+4 n+8\right)+\alpha \gamma+\varepsilon\right]}{8 \delta(n+2)\left[4 \beta a\left(n^{2}+4 n+8\right)-\alpha\right]}\right\}^{\frac{1}{n}}, k=n \sqrt{a}, c=\frac{\delta A^{n} n^{4}}{8 \beta k^{4}(n+1)(n+2)(3 n+4)(n+4)}+\frac{\lambda}{\beta}$ and $a=\frac{(\lambda-\beta \gamma)\left(n^{2}+4 n+8\right)+\sqrt{(\beta \gamma-\lambda)^{2}\left(n^{2}+4 n+8\right)^{2}+16(\alpha \lambda+\beta \varepsilon)(\alpha \gamma+\varepsilon)(n+2)^{2}}}{(\alpha \lambda+\beta \varepsilon)(n+2)^{2}}$.

Note that the equation (1) possesses the mass and momentum conserved quantities that are respectively given by

$$
M=\int_{-\infty}^{+\infty} u(x, t) d x=\frac{A \Gamma\left(\frac{1}{2}\right) \Gamma\left(\frac{2}{n}\right)}{k \Gamma\left(\frac{1}{2}+\frac{2}{n}\right)},
$$


and

$$
P=\int_{-\infty}^{+\infty} u^{2}(x, t) d x=\frac{A^{2} \Gamma\left(\frac{1}{2}\right) \Gamma\left(\frac{4}{n}\right)}{k \Gamma\left(\frac{1}{2}+\frac{4}{n}\right)},
$$

where $\Gamma(t)$ represents gamma function that is defined as

$$
\Gamma(t)=\int_{0}^{+\infty} e^{x} x^{t-1} d x
$$

\section{Tanh Ansatz Method}

Similar of the sech ansatz method, we assume a solitary wave ansatz of the following form (Razborova, Ahmed, \& Biswas, 2014; Triki, \& Wazwaz, 2009; Biswas, 2009):

$$
u(x, t)=A \tanh ^{p} \xi .
$$

where $\xi=k(x-c t)$, and the parameters $A, k, c, p$ will be determined later. From (20), we obtain

$$
\begin{gathered}
u_{t}=A k c p\left(\tanh ^{p+1} \xi-\tanh ^{p-1} \xi\right), \\
u_{x}=-A k p\left(\tanh ^{p+1} \xi-\tanh ^{p-1} \xi\right), \\
u^{n} u_{x}=-A^{n+1} k p\left[\tanh ^{(n+1) p+1} \xi-\tanh ^{(n+1) p-1} \xi\right], \\
u_{x x t}=-A k^{3} c p\left[(p-1)(p-2) \tanh ^{p-3} \xi-\left(3 p^{2}-3 p+2\right) \tanh ^{p-1} \xi\right. \\
\left.+\left(3 p^{2}+3 p+2\right) \tanh ^{p+1} \xi-(p+1)(p+2) \tanh ^{p+3} \xi\right], \\
u_{x x x}=A k^{3} p\left[(p-1)(p-2) \tanh ^{p-3} \xi-\left(3 p^{2}-3 p+2\right) \tanh ^{p-1} \xi\right. \\
\left.+\left(3 p^{2}+3 p+2\right) \tanh ^{p+1} \xi-(p+1)(p+2) \tanh ^{p+3} \xi\right], \\
-(p+1)(p+2)(p+3)(p+4) \tanh ^{p+5} \xi-5(p-1)(p-2)\left(p^{2}-3 p+4\right) \tanh ^{p-3} \xi \\
+5(p+1)(p+2)\left(p^{2}+3 p+4\right) \tanh ^{p+3}+2\left(5 p^{4}-10 p^{3}+25 p^{2}-20 p+8\right) \tanh ^{p-1} \xi \\
\left.-2\left(5 p^{4}+10 p^{3}+25 p^{2}+20 p+8\right) \tanh ^{p+1} \xi\right] \\
+(p+1)(p+2)(p+3)(p+4) \tanh ^{p+5} \xi-5(p-1)(p-2)\left(p^{2}-3 p+4\right) \tanh ^{p-3} \xi \\
u_{x x x x}=A k^{5} p\left[(p-1)(p-2)(p-3)(p-4) \tanh ^{p-5} \xi\right. \\
\left.-2\left(5 p^{4}+10 p^{3}+25 p^{2}+20 p+8\right) \tanh ^{p+1} \xi\right] .
\end{gathered}
$$

Substituting (21)-(27) into (1) gives

$$
\begin{array}{r}
A k p\left[\gamma-c-k^{2}(\alpha c+\varepsilon)\left(3 p^{2}-3 p+2\right)+2 k^{4}(\lambda-\beta c)\left(5 p^{4}-10 p^{3}\right.\right. \\
\left.\left.+25 p^{2}-20 p+8\right)\right] \tanh ^{p-1} \xi+A k p\left[c-\gamma+k^{2}(\alpha c+\varepsilon)\left(3 p^{2}+3 p+2\right)\right. \\
\left.+2 k^{4}(\beta c-\lambda)\left(5 p^{4}+10 p^{3}+25 p^{2}+20 p+8\right)\right] \tanh ^{p+1} \xi \\
+A k^{3} p(p-1)(p-2)\left[\alpha c+\varepsilon+5 k^{2}(\beta c-\lambda)\left(p^{2}-3 p+4\right)\right] \tanh ^{p-3} \xi \\
-A k^{3} p(p+1)(p+2)\left[\alpha c+\varepsilon+5 k^{2}(\beta c-\lambda)\left(p^{2}+3 p+4\right)\right] \tanh ^{p+3} \xi \\
-A k^{5} p(p-1)(p-2)(p-3)(p-4)(\beta c-\lambda) \tanh ^{p-5} \xi \\
+A k^{5} p(p+1)(p+2)(p+3)(p+4)(\beta c-\lambda) \tanh ^{p+5} \xi \\
-\delta A^{n+1} k p\left[\tanh ^{(n+1) p+1} \xi-\tanh ^{(n+1) p-1} \xi\right]=0 .
\end{array}
$$


Equating the exponents of $\tanh ^{(n+1) p+1} \xi$ and $\tanh ^{p+3} \xi$ terms in (28), we get

$$
(n+1) p+1=p+3,
$$

which yields the following analytical condition

$$
p=\frac{2}{n} .
$$

For simplicity, we only discuss the following two cases of $n=1$ and $n=2$.

\subsection{Case $1: n=1$}

The equation (1) becomes the Rosenau-Kawahara-RLW equation:

$$
u_{t}-\alpha u_{x x t}+\beta u_{x x x x t}+\gamma u_{x}+\delta u u_{x}+\varepsilon u_{x x x}+\lambda u_{x x x x x}=0 .
$$

For $n=1$, substituting (30) into (28), setting the coefficients of $\tanh ^{j} \xi(j= \pm 1, \pm 3,5,7)$ to zero, we obtain a dark 1 -soliton solution of the Rosenau-Kawahara-RLW equation (31):

$$
u(x, t)=A \tanh ^{2}[k(x-c t)]
$$

where $A=\frac{3(\lambda-\beta \gamma)}{2 \beta \delta}, k=\sqrt{\frac{\beta \gamma-\lambda}{8(\alpha \lambda+\beta \varepsilon)}}$ and $c=\frac{\lambda}{\beta}$.

3.2 Case $2: n=2$

The equation (1) becomes the modified Rosenau-Kawahara-RLW equation:

$$
u_{t}-\alpha u_{x x t}+\beta u_{x x x x t}+\gamma u_{x}+\delta u^{2} u_{x}+\varepsilon u_{x x x}+\lambda u_{x x x x x}=0 .
$$

For $n=2$, substituting (30) into (28), setting the coefficients of $\tanh ^{j} \xi(j=0, \pm 2, \pm 4,6)$ to zero, we obtain a dark 1 -soliton solution of the modified Rosenau-Kawahara-RLW equation (33):

$$
u(x, t)=A \tanh [k(x-c t)] .
$$

where $A=\sqrt{\frac{3(\lambda-\beta \gamma)}{\beta \delta}}, k=\sqrt{\frac{\beta \gamma-\lambda}{2(\alpha \lambda+\beta \varepsilon)}}$ and $c=\frac{\lambda}{\beta}$.

\section{Discussion}

In this paper, we apply sech ansatz method and tanh ansatz method to derive exact bright and dark 1-soliton solutions of the general Rosenau-Kawahara-RLW equation. In addition, more explicit solutions for the general Rosenau-Kawahara-RLW equation can be obtain by other techniques, such as tanh-coth method, exp-function method and $G^{\prime} / G$-expansion method, and so on.

\section{Acknowledgements}

The referees have reviewed the paper very carefully. The authors express their deep thanks for the comments.

\section{References}

Benjamin, T. B., Bona J. L., \& Mahony, J. J. (1972). Model equations for long waves in nonlinear dispersive systems. Philos. Trans. Roy. Soc. London Ser. A, 272, 47-78. http://dx.doi.org.sci-hub.org/10.1098/rsta.1972.0032

Biswas, A. (2009). 1-soliton solution of the $B(m, n)$ equation with generalized evolution. Commun. Nonlinear Sci. Numer. Simul., 14, 3226-3229. http://dx.doi.org/10.1016/j.cnsns.2008.12.025

Esfahani, A. (2011). Solitary wave solutions for generalized Rosenau-KdV equation. Commun. Theor. Phys., 55, 396-398.

Korteweg, D. J. \& Vires, G. de. (1895). On the change of form of long waves advancing in a rectangular channel, and a new type of long stationary wave. Phil. Mag, 539, 422-443. http://dx.doi.org/10.1080/14786449508620739

Razborova, p., Ahmed, B., \& Biswas, A. (2014). Solitons, shock waves and conservation laws of Rosenau-KdVRLW equation with power law nonlinearity. Appl. Math. Inf. Sci., 8, 485-491. http://dx.doi.org/10.12785/amis/080205 
Rosenau, P. (1988). Dynamics of dense discrete systems. Progr. Theor. Phys., 79, 1028-1042. http://dx.doi.org.scihub.org/10.1143/PTP.79.1028

Triki, H. \& Wazwaz, A. M. (2009). Bright and dark soliton solutions for a $K(m, n)$ equation with $t$-dependent coefficients. Phys. Lett. A, 373, 2162-215. http://dx.doi.org.sci-hub.org/10.1016/j.physleta.2009.04.029

Zuo, J. M. (2009). Solitons and periodic solutions for the Rosenau-KdV and Rosenau-Kawahara equation. Appl. Math. Comp., 215, 835-840. http://dx.doi.org/10.1016/j.amc.2009.06.011

\section{Copyrights}

Copyright for this article is retained by the author(s), with first publication rights granted to the journal.

This is an open-access article distributed under the terms and conditions of the Creative Commons Attribution license (http://creativecommons.org/licenses/by/3.0/). 